\title{
Thermally diffused expanded-core fibers with gain or loss
}

\author{
George S. Kliros* \\ Department of Aeronautical Sciences, Division of Electronics and Communication Engineering \\ Hellenic Air-Force Academy, Dekeleia Attica GR-1010, Greece
}

Received July 17, 2009; accepted September 28, 2009; published September 30, 2009

\begin{abstract}
We investigate the modal properties of thermally diffused expanded-core fibers (TEC) with loss or gain. The corresponding Helmholtz equation with complex refractive index profile is solved numerically using Galerkin's method. The imaginary part of the modal electric field results in wave-front distortion showing that the power flows out or into the doped region according to the sign of the imaginary part of the refractive index. The spectral characteristics of the calculated gain or loss are discussed. Our results may potentially be useful to design high power fiber lasers or amplifiers using TEC technique.
\end{abstract}

Thermally diffused expanded-core (TEC) fiber (Fig.1) can have very low insertion loss [1-3] and solve alignment problems of connecting two single mode fibers (SMFs) with different core sizes or coupling a SMF to a laser diode, and also of assembling micro-optic components [4,5]. Moreover, TEC fibers have been recently used for the design of band-edge filters with a high cut-off property or tunable comb-filters [6,7]. A TEC fiber has an enlarged mode field radius (MFR) obtained by heating a conventional single-mode fiber locally at a high temperature $\left(\sim 1300-1650{ }^{\circ} \mathrm{C}\right)$ and diffusing some dopants as Ge or F into the core. The core expansion rate depends on the heating temperature, the heating time and the dopant intensity in the fiber core [8]. TEC fiber has the feature that although thermal diffusion changes the refractive-index profile, the normalized frequency does not change and hence the single mode condition is maintained through the process. Recently, TEC-fiber has been proposed to suppress stimulated Brillouin scattering in high power fiber lasers or amplifiers. Compared to conventional large mode area fiber, a total $7 \mathrm{~dB}$ suppression is achieved [9].

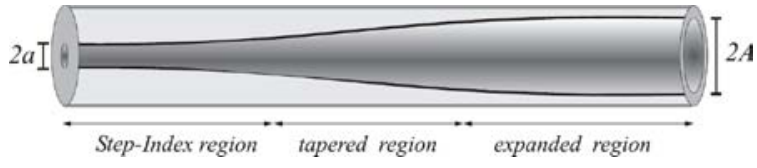

Fig. 1. Schematic illustration of a thermally expanded core fiber.

The critical characteristics in fiber lasers or amplifiers are the gain and loss along the length of the fiber. Except for models based on rate and propagation equations, an alternative method is to analyze optical fibers whose

*E-mail: gsksma@hol.gr refractive-index profile is described in terms of a complex function. The gain or loss can be described by the imaginary component of the complex propagation constant, which is critically dependent on the imaginary component of the complex refractive-index profile.

In this letter, we analyse, for the first time to our knowledge, the modal characteristics of TEC fibers with gain or loss. Under the weakly guiding approximation, the scalar Helmholtz eigenvalue equation, for a given azimuthal mode number $m$, can be written in polar coordinates as

$$
\frac{d^{2} E(r)}{d r^{2}}+\frac{1}{r} \frac{d E(r)}{d r}+\left(k^{2} n^{2}(r)-\beta^{2}-\frac{m^{2}}{r^{2}}\right) E(r)=0
$$

where $E=E(r)$ represents the radial variation of the modal field, $k$ is the free-space wavenumber and $\beta$ is the propagation constant. The refractive index profile $n(r)$ of the TEC fiber can be successfully approximated by $[8,10]$ :

$$
n^{2}(r)=n_{c l}^{2}+\frac{\alpha^{2}}{A^{2}}\left(n_{c o}^{2}-n_{c l}^{2}\right) e^{-\frac{r^{2}}{A^{2}}}
$$

where $A$ is the core radius of the fiber after the heat treatment, $n_{c o}$ and $n_{c l}$ are the refractive indexes of the core and the cladding of a step-index fiber, before the heat treatment, respectively. The expanded core radius is given in terms of diffusion coefficient of the dopant $D$ and the heating time $t$ as $A=2 \sqrt{D t}$.

As we are dealing with a structure with loss or gain, we consider in (2) a complex index core: $n_{c o}=n_{c o, r}+i n_{c o, i}$ and therefore the refractive index of the TEC fiber with gain or loss can be written in complex form as

$$
n^{2}(r)=\operatorname{Re}\left[n^{2}(r)\right]+i \operatorname{Im}\left[n^{2}(r)\right]
$$

Consequently, the scalar Helmholtz eigenvalue equation becomes non-Hermitian, meaning the eigenvalues may be complex. We thus expect the propagation constant to have both real and imaginary part, writing $\beta=\beta_{r}+\mathrm{i} \beta$. If $\beta$ is positive the solutions will 
decay exponentially correspon-ding to loss in optical power, whereas a negative value of $\beta$ i results in solutions increasing in power along the fiber length, corresponding to amplification.

We apply Galerkin's numerical method in cylindrical coordinates to find the modal field of the TEC fiber with complex refractive index profile [11]. The main advantage of the method is its versatility and robustness in treating circular fibers with arbitrary index profiles. The complex modal field is expanded in a set of associated Laguerre-Gauss basis functions

$$
E(R)=\sum_{i=0}^{N-1} a_{i} \varphi_{i}^{m}(R)
$$

where

$$
\varphi_{i}^{m}(R)=\sqrt{\frac{i !}{(i+m) !}} e^{-m / 2} e^{-R^{2} / 2} L_{i}^{m}(R), \quad i=0,1, \ldots
$$

$L_{i}^{m}(R)$ are the associated Laguerre polynomial and $N$ is the number of basis functions used. The normalized parameter $R$ is defined as $R=\sigma r^{2} / \alpha^{2}$ where $\sigma$ is an arbitrary positive number that affects the convergence, accuracy and computational time [5]. Then, Equation (1) is transformed into a matrix eigenvalue equation for the propagation constant as $\boldsymbol{H a}=\beta^{2} \mathbf{a}$, where the elements of the complex $N x N$ matrix $\boldsymbol{H}$, are given by

$$
H_{i j}=-\frac{\sigma}{\alpha^{2}} T_{i j}+k^{2} I_{i j}
$$

where

$$
\begin{gathered}
T_{i j}=(2 j+m+1) \delta_{i j}+\sqrt{(j+1)(j+m+1)} \delta_{j+1, i} \\
+\sqrt{j(j+m)} \delta_{j-1, i} \\
I_{i j}=\int_{0}^{\infty} n^{2}(\sqrt{R / \sigma}) \varphi_{i}^{m} \varphi_{j}^{m} R d R
\end{gathered}
$$

The eigenvector $\mathbf{a}$ is a column vector that contains the complex coefficients $a_{i}$. In our calculations, the parameter $\sigma$ is chosen equal to the expanded core radius $A$ of the TEC fiber and the complex eigenvalue problem is solved using optimized EISPACK matrix eigensystem routines [12]. As a final result, the complex propagation constant $\beta$ of a guided mode is obtained and the gain or loss of the propagating signal power, in decibels per meter, is given by $g(d B / m)=20 \log (e) \operatorname{Im}(\beta)$.

The TEC fiber under consideration has a thermally expanded core with radius $\mathrm{A}=13 \mu \mathrm{m}$ which corresponds to $10 \mathrm{~h}$ of thermal diffusion of $\mathrm{GeO}_{2}$ atoms into the core of a standard SMF-28 fiber at $1300^{\circ} \mathrm{C}$ [8]. We found that only the fundamental mode $\left(\mathrm{LP}_{01}\right)$ exists at wavelength $\lambda=1.55 \mu \mathrm{m}$ and the mode field radius of the fiber has been expanded to $20 \mu \mathrm{m}$. Such SMF with over-expanded core can be used for designing stimulated Brillouin scattering suppression fiber-amplifiers [9].

Figures 1(a) and 1(b) show the real and imaginary part of the fundamental mode, respectively, for three different values of the imaginary part $n_{c o, I}$ at wavelength $\lambda=1.55$ $\mu \mathrm{m}$. The imaginary part of the modal electric field is a result of the imaginary part of the refractive index introduced into the fiber core. As it is seen, the imaginary parts of the modal fields in Fig. 1(b) are overlapped after multiplication by 10 for $n_{c o, i}=10^{-5}$ and by 100 for $n_{c o, i}=$ $10^{-6}$. An apparent characteristic of the imaginary part of the modal field is that the field reaches the minimum at a radial distance equal to the mode field radius of the fundamental mode $(\sim 20 \mu \mathrm{m})$.

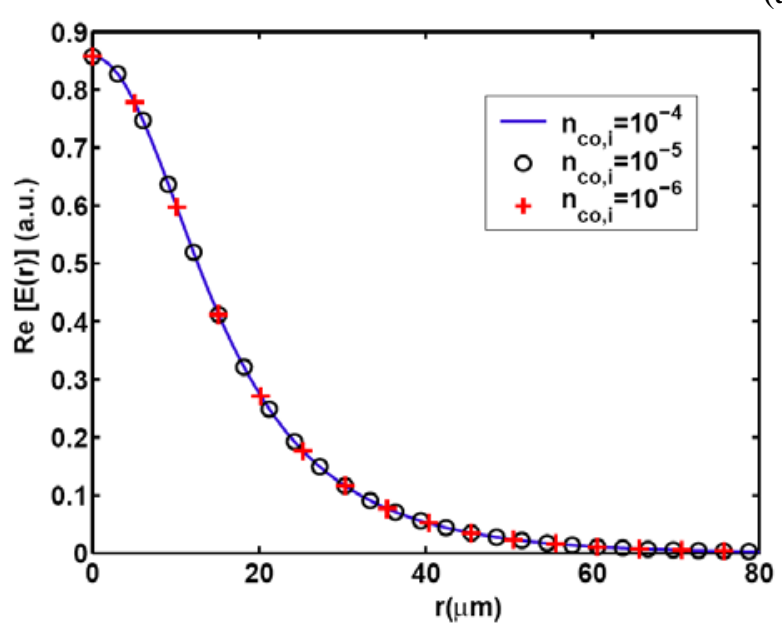

(b)

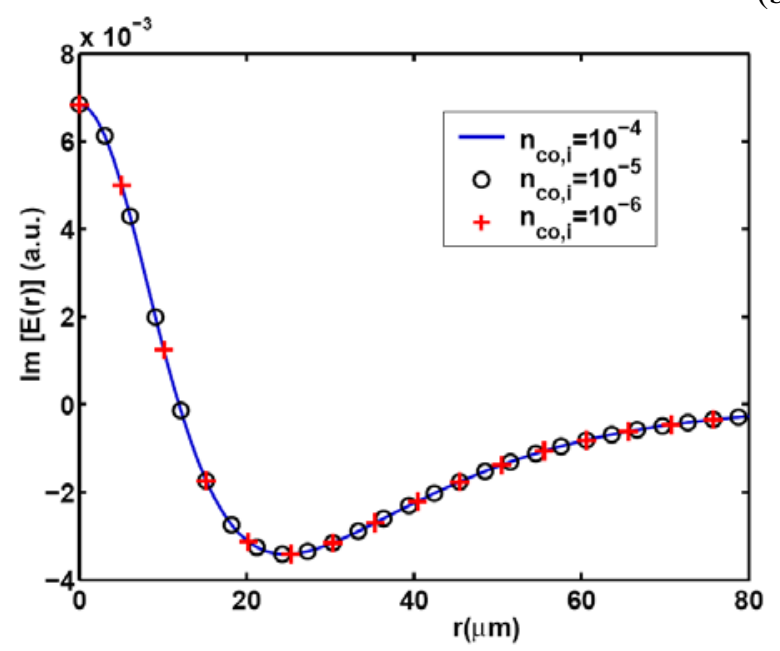

Fig. 1. Real (a) and imaginary (b) part of the modal field of complex refractive index TEC-fiber. 
Figure 2 shows the phase distribution in the fiber's cross section for three experimentally acceptable values $n_{c o, i}$ at $\lambda=1.55 \mu \mathrm{m}$. This phase can be considered to be the wave-front of the fundamental mode $\mathrm{LP}_{01}$. It is well known that the wave-front is a plane for ideal fibers (no loss or gain), but when the imaginary part of the refractive index is introduced, the wave-front is distorted. The phase distortion revealed in Fig. 2 shows that there is a power outflow from the doped region to cladding, which is induced by the axial gain. We find that the phase distortion is principally dominated by the imaginary part of the refractive index $n_{c o, i}$. Note that the phase is multiplied by 10 for $n_{c o, i}=10^{-5}$ and 100 for $n_{c o, i}=10^{-6}$. It is bvious that these curves are overlapped and therefore the phase is analogue to $n_{c o, i}$.

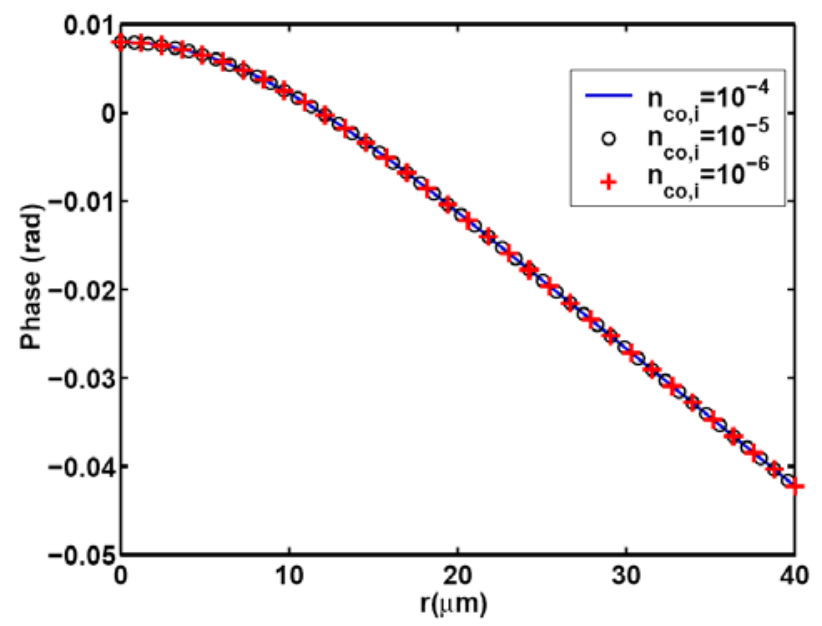

Fig. 2. Radial phase distribution at $\lambda=1.55 \mu \mathrm{m}$ for three different values of $n_{c o, i}$. The phase has been multiplied by 10 for $n_{c o, i}=10^{-5}$ and by 100 for $n_{c o, i}=10^{-6}$.

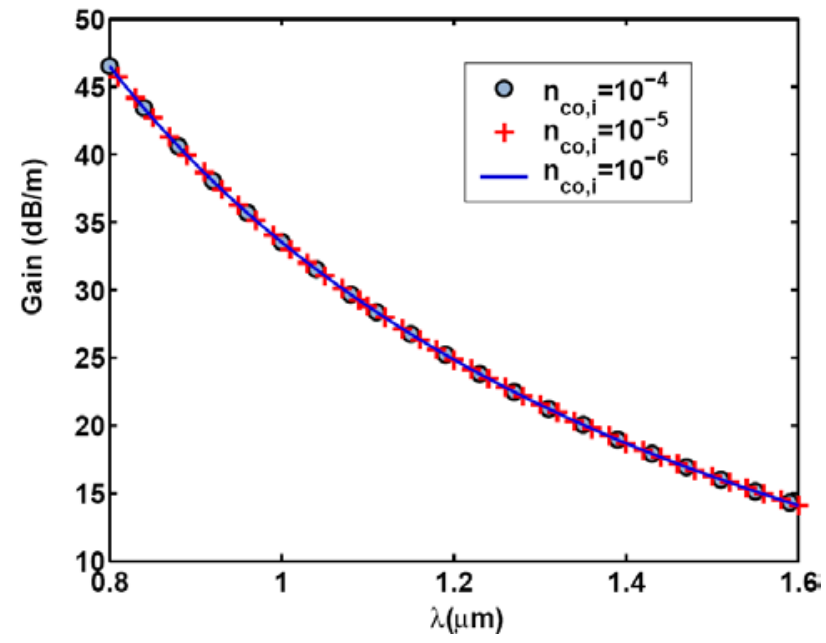

Fig. 3. Gain versus operating wavelength. The gain has been divided by 10 for $n_{c o, i}=10^{-4}$ and multiplied by 10 for $n_{c o, i}=10^{-6}$.
Figure 3 shows the gain (or loss) in $\mathrm{dB} / \mathrm{m}$, as a function of wavelength for three different values of $n_{c o, i}$. As it is seen, when the gain corresponding to $n_{c o, i}=10^{-4}$ is divided by 10 and the gain corresponding to $n_{c o, i}=10^{-6}$ multiplied by 10 , the curves are overlapped and therefore the gain or loss is analogue to $n_{c o, i}$. Moreover, the gain increases as the wavelength decreases because more field energy is confined in the high-index region.

In conclusion, the modal characteristics of TEC fibers with gain or loss are analysed by Gelerkin's method. The imaginary part of the electric field results in wave-front distortion in the fundamental mode of the TEC fiber with loss or gain, which means that there is a power flow out or into the doped region according to the sign of the imaginary part of the refractive index. It can be assumed that the modal field is a combination of some normal modes of the corresponding fiber without gain or loss and therefore, induces a wave-front distortion. The spectral characteristics of gain are also examined for three experimentally acceptable values of the imaginary part of the core-refractive index. As the operating wavelength $\lambda$ increases, the gain decreases because the optical power confined to the core decreases. Our results may potentially be useful to design high power fiber lasers or amplifiers using TEC technique.

\section{References}

[1] K. Shiraishi, Y. Aizawa and S. Kawakami, J. Lightwave Technol. 8, 1151 (1990).

[2] T. Haibara, T. Nakashima, M. Matsumoto, H. Hanafusa, IEEE Photonics Techn. Lett. 3(4), 348 (1991).

[3] M. Kihara, M. Matsumoto, T. Haibara, S. Tomita , J. Lightwave Techn. 14(10), 2209 (1996).

[4] G. S. Kliros, P. C. Divari, J. Mater. Sci: Mater. Electron. 20, S59-S62 (2009).

[5] P. C. Divari, G. S. Kliros, Optik 120, 222 (2009).

[6] K. Kima, K. Leea, E. Shina, H. Songa, K. Honga, S. Hwangboa, K. Sohnb, Opt. Comm. 180, 51 (2006).

[7] L. V. Nguyen, D. Hwang, D. S. Moon, Y. Chung, Optics Comm. 281, 5793 (2008).

[8] G. S. Kliros, N. Tsironikos, Optik 116, 365 (2005).

[9] A. Liu, Opt. Express 15, 977 (2007). http://www.opticsinfobase.org/oe/abstract.cfm?URI=0e-15-3-977

[10] S. Savovic, A. Djordjevich, Optical Materials 30, 1427 (2008).

[11] G. S. Kliros, P. C. Divari, Proc. of $14^{\text {th }}$ Micro-optics Conf. MOC'08, Brussels, Belgium, p. 135

[12] B. T. Smith, J. M. Boyle, J. J. Dongarra, B. S. Garbow, Y. Ikebe, V. C. Klema and C. B. Moler, Matrix Eigensystem Routines-EISPACK Guide, Lecture Notes in Computer Science 6, ed. G. Goos and J. Hartmanis (Springer, Berlin, 1976). 